\title{
Significance of complete right bundle-branch block when an isolated finding An echocardiographic study
}

\author{
MARK DANCY, ^ GRAHAM LEECH, AUBREY LEATHAM \\ From the Cardiac Department, St George's Hospital, London
}

SUMMARY Twenty-seven patients with complete right bundle-branch block as the only abnormal finding were studied using high speed $M$-mode echocardiography to determine the effect of the electrical delay on the mechanical events of right ventricular systole. Pulmonary valve opening (PVOm) was delayed in all cases. In some the delay was mainly between mitral valve closure (MVC) and tricuspid valve closure (TVC), and this was designated proximal block. In the others the main delay was between tricuspid valve closure and pulmonary valve opening and this was designated distal block. The patients were divided into those with proximal and those with distal block by calculating the ratio TVC-PVOm/MVC-TVC. Twelve out of 13 of those with distal delay but only one out of 14 of those with proximal delay had episodes of syncope or near syncope. These results are consistent with previous theories about the pathophysiology of right bundle-branch block. Echocardiography may offer a non-invasive method to estimate the prognosis in isolated right bundle-branch block.

When complete bundle-branch block is discovered as an isolated finding in a patient who has no other evidence of heart disease, the long-term prognosis is difficult to assess, and this is particularly important in aviation and insurance medicine. The finding of right bundle-branch block is usually unimportant, ${ }^{1}$ but in occasional patients it is an early sign of diffuse conducting tissue disease, cardiomyopathy, or coronary artery disease. There is at present no reliable way of distinguishing this important second group of patients with underlying or progressive disease.

It is now fairly well established that there are two forms of right bundle-branch block: one in which there is interruption of the main right branch of the bundle of His, termed proximal block, and the other in which the terminal ramifications of the right bundle are affected, termed distal or arborisation block..$^{2-4}$ Brooks et al. ${ }^{5}$ suggested that patients with right bundle-branch block could be divided into those with proximal block and those with distal block by timing right sided systolic events using echophonocardiography; these authors speculated that there might be a difference in prognosis between the two groups, prox-

\footnotetext{
*Jensen Research Fellow, St George's Hospital.

Accepted for publication 21 May 1982
}

imal being relatively isolated and benign, but distal being associated with diffuse disease which was likely to be more widespread and progressive. In the group thought to have proximal block, they found that the right sided delay was confined to prolongation of the time interval between mitral valve closure and tricuspid valve closure on the echocardiogram. In the group thought to have distal block the delay was mainly between tricuspid valve closure and pulmonary valve opening.

In order to test the hypothesis that proximal block is usually a benign condition, and that distal block is more likely to be associated with more widespread disease, we measured these time intervals in patients in whom right bundle-branch block was an isolated finding at a routine examination, and compared them with the results from an otherwise identical group of patients with right bundle-branch block who had a history of syncope or near syncope.

\section{Patients and method}

The 27 patients included in this study all had basic sinus rhythm with typical complete right bundlebranch block at the time of study. None had evidence of any other cardiac disorder on radiographic, echocardiographic, or electrocardiographic grounds. 
In particular there was no electrocardiographic evidence of left sided conduction disease (normal PR interval and QRS axis). In 14 patients the right bundle-branch block had been discovered at a routine examination (for example for a pilot's licence or insurance) and they were free of symptoms. The remaining 13 patients had suffered attacks of syncope or near syncope though they were otherwise symptom free. Eleven of the 13 had had documented episodes of complete heart block. Eight of them had had demand pacemakers fitted. Of those with pacemakers, three were in sinus rhythm inhibiting the pacemaker at the time of the study, and in five sinus rhythm was induced by external inhibition of the pacemaker using surface electrodes and an impulse of $100 /$ min from an external pacing unit.

There was no significant difference in age between those patients with symptoms (mean 66 years, range 42 to 83 ) and those without (mean 60 years, range 42 to 69 ). The results were compared with those from a group of 63 normal subjects who were younger than the patients, with a mean age of 33 years (range 19 to 56 years).

Echocardiograms were recorded on a Smith Kline Ultrasonoscope using a $2 \cdot 25 \mathrm{MHz}$ focused transducer with pulse repetition frequency increased to 2000/s. The output was displayed on a Cambridge 6 channel recorder with maximum paper speed increased to 200 $\mathrm{mm} / \mathrm{s}$. Magnified recordings were made of the mitral, tricuspid, and pulmonary valves, together with a high frequency phonocardiogram from the fourth left intercostal space and the standard electrocardiographic lead showing the earliest onset of QRS. The technique and details of measurement have been fully described elsewhere. ${ }^{5}$ Measurements were made from $\mathrm{Q}$ to mitral valve closure (MVC), $\mathrm{Q}$ to tricuspid valve closure (TVC), and $Q$ to pulmonary valve opening (PVOm) (maximum pulmonary valve opening was measured instead of the onset of pulmonary valve opening as it is a reproducible event that can be measured with great accuracy). From these results the time intervals MVC to TVC and TVC to PVOm were calculated.

Statistical analysis was carried out using Student's unpaired $t$ test unless otherwise stated.

\section{Results}

Mean time interval measurements for the two patient groups and the normal subjects are shown in the Table. The results for individual subjects are shown graphically in Fig 1; values of MVC-TVC (interrupted line) and TVC-PVOm (solid line) for each subject are indicated with reference to the time of TVC. Heavy lines under each patient group show mean values for that group and only the mean values are given for the 63 normal subjects.
Table Mean time intervals in 63 normal subjects, 14 patients with right bundle-branch block and no symptoms, and 13 patients with right bundle-branch block and syncope ( $p$ values refer to comparisons between normals and patient groups)

\begin{tabular}{lllll}
\hline Subjects & No. & Q-MVC & MVC-TVC & TVC-PVOm \\
\hline Normal & 63 & $55 \pm 11$ & $34 \pm 10 \cdot 5$ & $39 \pm 12$ \\
Without symptoms & 14 & $57 \pm 22 \dagger$ & $58 \pm 11^{\star \star}$ & $53 \pm 12^{\star}$ \\
With symptoms & 13 & $59 \pm 15 \dagger$ & $33 \pm 18 \dagger$ & $95 \pm 32^{\star \star}$ \\
\hline
\end{tabular}

All figures in $\mathrm{ms} \pm \mathrm{SD}$.

tNS;

${ }_{\star}^{*} \mathrm{p}<0.005$;

$\star \star \mathrm{p}<0.001$

Q-MVC, interval from onset of QRS to mitral valve closure; MVC-TVC, interval from mitral to tricuspid valve closure; TVC-PVOm, interval from tricuspid valve closure to maximal pulmonary valve opening.

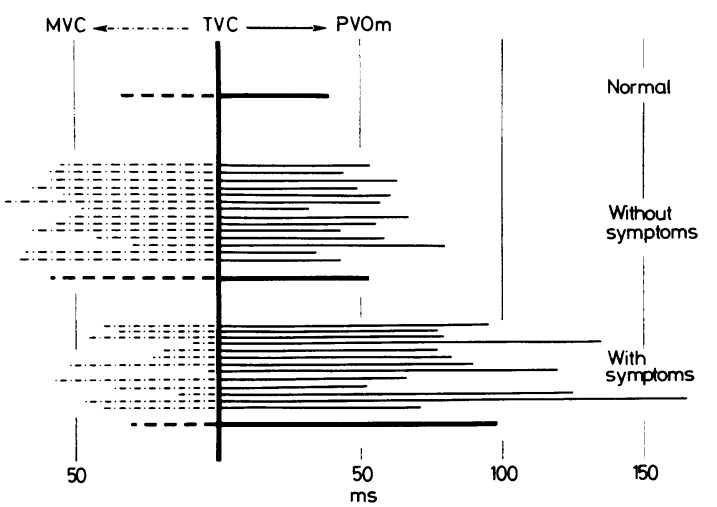

Fig. 1 Values of the intervals of MVC-TVC (interrupted horizontal lines to the left of the vertical line), and TVC-PVOm (solid horizontal lines to the right of the vertical line) displayed in relation to the time of tricuspid valve closure (the vertical line). Beneath each patient group is the mean (thicker lines).

MVC TO TVC INTERVAL

In all but one of the 14 symptom-free patients with right bundle-branch block the time interval from mitral valve closure to tricuspid valve closure was longer than the mean of the normal subjects. This had been strongly suspected clinically in most because of wide splitting of the first heart sound. In addition, the mean value was significantly prolonged in these symptom-free patients when compared either with those with symptoms $(p<0.001)$ or with normal subjects $(p<0.001)$.

In the patients with syncope the mean interval was not prolonged, but there was considerable individual variation (Fig 2).

TVC TO PVOm INTERVAL

In the symptom-free patients the time interval from tricuspid valve closure to pulmonary valve opening was within the normal range in 10, but in four it was 


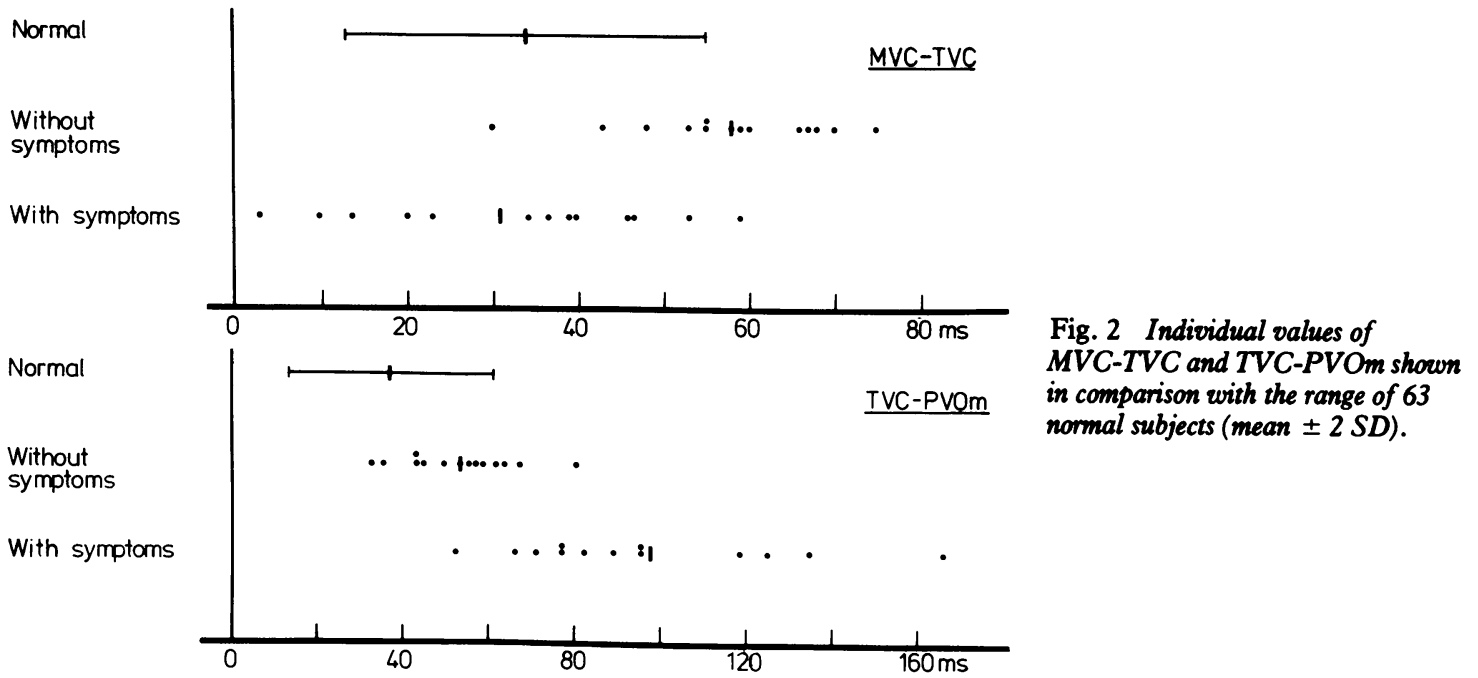

slightly prolonged, and the mean was slightly greater than normal $(p<0.005)$. It should be noted in this respect that both groups of patients were significantly older than the normal subjects. In all but one of the patients with syncope, TVC-PVOm was abnormally prolonged, and was usually greatly prolonged. Mean TVC-PVOm was significantly increased in these patients both when compared with the normal subjects $(p<0.001)$ as well as with the symptom-free group $(p<0.001)$.

\section{RATIO OF TVC-PVOm/MVC-TVC (Fig 3)}

By combining the two measurements a greater separation was achieved between the patients with symptoms and those without. This ratio was greater than the arbitrary value 1.4 in all but one of the patients with syncope, and less than 1.4 in all but one of the symptomless patients with right bundle-branch block $\left(\mathrm{p}<0.001\right.$ by $\chi^{2}$ test $)$.

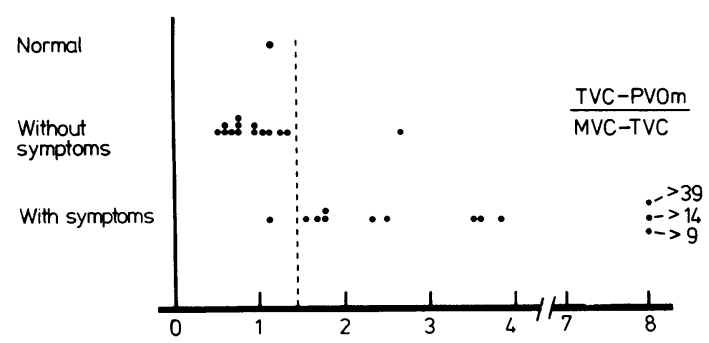

Fig. 3 Ratio of TVC-PVOm/MVC-TVC for all the patients. An arbitrary line is drawn at 1.4. Those patients with values below this are considered to have proximal block, whereas those with values above are considered to have distal block.

\section{Discussion}

There is considerable evidence from physiological studies to suggest that the electrocardiographic pattern of right bundle-branch block is identical whether the interruption is in the proximal or distal portions of the right His-Purkinje network. ${ }^{6-9}$ Anatomically, the right bundle comprises a single group of fibres which arborises only at the periphery. Interruption of the proximal portion is therefore likely to cause delay in the onset of right ventricular contraction, but, assuming the distal network is intact, contraction should then proceed normally. On the other hand, disease affecting the peripheral branches, but not the proximal bundle, ought to cause asynchronous contraction, slowing the overall rate of rise of pressure without delaying its onset. In both cases the effect would be to delay the opening and closure of the pulmonary valve. Generalised disease of the right conduction system should cause proximal and distal delays in the same patient.

Animal studies with selective interruption of the proximal $^{67}$ and distal $^{89}$ fibres have confirmed these suggestions. In man the relation between the anatomical site of interruption of the right bundle and the consequent electrical delays have been studied by epicardial isochrone mapping during surgical procedures that produced right bundle-branch block. ${ }^{10}$ Proximal interruption produced similar delays in activation of all parts of the right ventricle, whereas distal interruption slowed the rate of propagation of the depolarisation wave.

Alterations in the sequence of events during systole are reflected in the heart sounds. Indeed, a study of the sequence of the aortic and pulmonary components 
of the second heart sound provided the first clue that the early electrocardiographers had confused the electrocardiographic patterns of right and left bundlebranch block which had been derived from studies on dogs. ${ }^{11}$ Abnormally wide splitting of the first heart sound, attributed to delay of tricuspid valve closure, was found to be a feature of apparently isolated right bundle-branch block, and was also found in other circumstances with delayed onset of right ventricular contraction, such as with pacing from the left ventricle or with ectopic beats of left ventricular origin. ${ }^{12}$ Echophonocardiographic studies in Ebstein's anomaly, in which the right bundle comprises only a short proximal group of fibres, showed a striking delay of the tricuspid component of the first heart sound.$^{13}$ It was pointed out, however, that the onset right ventricular systole was not invariably delayed in right bundle-branch block ${ }^{4}$ and an echocardiographic study confirmed that in some instances right bundlebranch block did not cause delay of tricuspid valve closure. ${ }^{5}$ These findings strongly suggested that in such cases the delay in activation was confined to the peripheral fibres. It was also noted that most of the patients in this group had cardiac abnormalities in addition to right bundle-branch block; indeed, peripheral delay would be expected with myocardial disease, myocardial infarction, and particularly idiopathic bundle-branch fibrosis which is the commonest cause of complete heart block. ${ }^{14-16}$

There is therefore substantial evidence that there are two pathologically distinct forms of isolated right bundle-branch block. Proximal block, caused by a single localised lesion, may be the underlying pathology in the majority of cases of isolated right bundlebranch block which statistically have an excellent prognosis. ${ }^{1}$ Distal block, where the underlying lesion is more diffuse, may be evidence of the progressive disease seen in a minority of patients. The present study was designed to show whether the position of the block could be predicted by echophonocardiographic timing studies, and whether this had any prognostic relevance. For this purpose, delay of tricuspid valve closure relative to mitral valve closure was regarded as evidence of proximal block, and delay between tricuspid valve closure and pulmonary valve opening as distal block.

The results for the symptom-free patients with right bundle-branch block showed that the mean value of MVC-TVC was significantly prolonged $(p<0.005$ compared both with normal subjects and with patients with symptoms) and in nine cases was outside the normal range. TVC-PVOm, however, was normal in the majority, though in four it was slightly prolonged. The patients with a history of syncope, whose electrocardiograms were indistinguishable from those of the symptom-free patients with right bundle-branch block, had striking prolongation of the mean TVC-PVOm interval, which was 2.4 times that of the normal subjects $(p<0.001$ compared with normal subjects and symptom-free patients). Most showed no delay of tricuspid valve closure, suggesting that the proximal portion of the right bundle was intact, but in some the interval from mitral to tricuspid valve closure was also prolonged, suggesting that is there was generalised disease affecting both proximal and distal regions.

Although there was some overlap of measured values for individuals in the two patient groups, this was reduced by expressing the intervals in the form of ratios (Fig. 3). An arbitrary division set at 1.4 for the ratio TVC-PVOm/MVC-TVC gave optimal separation, leaving only one symptom-free patient in the "distal section" and one patient with symptoms in the "proximal section".

Thus, the evidence for the separation of right $\vec{C}$ bundle-branch block into proximal and distal varieties $\mathbb{D}$ is based on electrophysiological, anatomical, and pathological findings, and we believe that it is possible to place patients in one of these two groups by means of echocardiography, or even by simple auscultation in many cases. Though the number of patients so far studied is small, this seems a promising technique for assessing the prognostic significance of right bundlebranch block. Only a long-term follow-up of patients with isolated right bundle-branch block will disclose whether or not distal block is more likely to be associated with progressive pathology.

\section{References}

1 Rotman M, Triebwasser JH. A clinical and follow-up study of right and left bundle branch block. Circulation 1975; 51: 477-84.

2 Oppenheimer BS, Rosthchild MA. Abnormalities in the QRS group of the electrocardiogram associated with 3 myocardial involvement. Proc Soc Exp Biol Med 1916; 14: 57-9.

3 Rosenman RH, Pick A, Katz LN. The electrocardiographic patterns and the localization of intraventricular $\mathrm{O}$ conduction defects. Am Heart f 1950; 40: 845-66.

4 Braunwald E, Morrow AG. Sequence of ventricular contraction in human bundle branch block. Am F Med 1957; 23: 205-11.

5 Brooks N, Leech G, Leatham A. Complete right bundle N branch block. Echophonocardiographic study of first $N$ heart sound and right ventricular contraction times. $B r \underset{\sigma}{\omega}$
Heart $\mathcal{J} 1979 ; 41: 637-46$.

6 Braun-Menéndez E, Solari LA. Asincronismo ventricu- $\bullet$ lar por sección de la ramas del haz de His; sección de la rama derecha. Rev Soc Argent Biol 1936; 12: 331-49.

7 Smith LA, Fields J, Kennamer R, Prinzmetal M. T Studies on the mechanism of ventricular activity. III: $O$ Contraction of the ventricles in experimental bundle branch block. Am Heart $\mathcal{F}$ 1952; 44: 231-7.

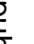


8 Abildskov JA, Eich RH, Harumi K, Smulyan $H$. Observations on the relation between ventricular activation sequence and the hemodynamic state. Circ Res 1965; 17: 236-47.

9 Millar K, Burgess MJ, Abildskov JA, Eich RH. Dependence of intraventricular pressure on activation order before and after experimental Purkinje net block. $\mathcal{F}$ Electrocardiol 1970; 3: 51-6.

10 Horowitz LN, Alexander JA, Edmunds LH Jr. Postoperative right bundle branch block: identification of three levels of block. Circulation 1980; 62: 319-28.

11 Wolferth CC, Margolies A. Asynchronism in contraction of the ventricles in the so-called common type of bundle branch block: its bearing on the determination of the side of the significant lesion and on the mechanism of split first and second heart sounds. Am Heart $\mathcal{F}$ 1935; 10: 425-52.

12 Haber E, Leatham A. Splitting of heart sounds from ventricular asynchrony in bundle-branch block, ven- tricular ectopic beats, and artificial pacing. $\mathrm{Br}$ Heart $\mathcal{f}$ 1965; 27: 691-6.

13 Crews TL, Pridie RB, Benham R, Leatham A. Auscultatory and phonocardiographic findings in Ebstein's anomaly. Correlation of first heart sound with ultrasonic records of tricuspid valve movement. $\mathrm{Br}$ Heart $\mathcal{F}$ 1972; 34: 681-7.

14 Zoob M, Shirley Smith K. The aetiology of complete heart block. Br Med F 1963; ii: 1149-54.

15 Lenégre J, Moreau P. Le block auriculoventriculaire chronique. Etude anatomique, clinique et histologique. Arch Mal Coeur 1964; 56: 867-88.

16 Davies M, Harris A. Pathological basis of primary heart block. Br Heart $\mathcal{f}$ 1969; 31: 219-26.

Requests for reprints to $\mathrm{Dr} C \mathrm{M}$ Dancy, Cardiac Department, St George's Hospital, London SW 17 ORE. 